\title{
Tanshinone I alleviates motor and cognitive impairments via suppressing oxidative stress in the neonatal rats after hypoxic- ischemic brain damage
}

\author{
Chunfang Dai ${ }^{1,2,3}$, Yannan Liu ${ }^{1,2,3}$ and Zhifang Dong ${ }^{1,2,3^{*}}$
}

\begin{abstract}
Neonatal hypoxia-ischemia is one of the main reasons that cause neuronal damage and neonatal death. Several studies have shown that tanshinone I (Tsl), one of the major ingredients of Danshen, exerts potential neuroprotective effect in adult mice exposed to permanent left cerebral ischemia. However, it is unclear whether administration of Tsl has neuroprotective effect on neonatal hypoxic-ischemic brain damage (HIBD), and if so, the potential mechanisms also remain unclear. Here, we reported that treatment with Tsl (5 mg/ $/ \mathrm{kg}$, i.p.) significantly alleviated the deficits of myodynamia and motor functions as well as the spatial learning and memory in the rat model of HIBD. These behavioral changes were accompanied by a significant decrease in the number of neuronal loss in the CA1 area of hippocampus. Moreover, ELISA assay showed that TsI significantly increased the production of antioxidants including total antioxidant capacity (T-AOC), glutathione (GSH), total superoxide dismutase (T-SOD) and catalase (CAT), and reduced the production of pro-oxidants including hydrogen peroxide $\left(\mathrm{H}_{2} \mathrm{O}_{2}\right)$, total nitric oxide synthase (T-NOS) and inducible nitric oxide synthase (iNOS). Taken together, these results indicate that Tsl presents potential neuroprotection against neuronal damage via exerting significantly antioxidative activity and against pro-oxidant challenge, thereby ameliorating hypoxia-ischemia-induced motor and cognitive impairments in the neonatal rats, suggesting that Tsl may be a potential therapeutic agent against HIBD.
\end{abstract}

Keywords: Hypoxic-ischemic brain damage, Tanshinone I, Learning and memory, Oxidative stress

\section{Introduction}

Neonatal hypoxic-ischemic encephalopathy (HIE), which is caused by perinatal hypoxia-ischemia, is one of the major reasons that lead to neuronal damage and neonatal death. The incidence of HIE is about 1 to 3 per 1000 term births $[1,2]$, and up to 40,000 to 50,000 infants are affected each year in China. The survivor exhibits motor disability or a variety of serious neurological sequela, such as cerebral palsy, epilepsy, severe learning impairment or

\footnotetext{
* Correspondence: zfdong@aliyun.com

'Ministry of Education Key Laboratory of Child Development and Disorders, Children's Hospital of Chongqing Medical University, 136 Zhongshan Er Road, Yuzhong District, Chongqing 400014, People's Republic of China ${ }^{2}$ Chongqing Key Laboratory of Translational Medical Research in Cognitive Development and Learning and Memory Disorders, Children's Hospital of Chongqing Medical University, 136 Zhongshan Er Road, Yuzhong District, Chongqing 400014, People's Republic of China

Full list of author information is available at the end of the article
}

intellectual deficiency [3-5], which diminish the quality of life of HIE children and increase the enormous burden of economic and spirit on their families and society. Therefore, the research for a potential neuroprotective therapy in order to guide the clinical treatment for HIE is particularly important.

Recent studies have proposed that oxidative injury to vital cellular structures contributes to the pathogenesis of HIE [6]. The potential mechanism underlying oxidative injury in HIE is that the neonatal brain is selectively vulnerable to oxidative stress, which results in altered reactive oxygen species metabolism [7] including the increased accumulation of hydrogen peroxide and subsequent neurotoxicity [8]. In addition, the brain of neonatal is immature and the nervous system exerts immature antioxidant defenses, which display less activity in antioxidant enzyme systems including superoxide 
dismutase (SOD) and glutathione peroxides [9, 10]. There is a growing body of evidence has shown that oxidative stress results in calcium mobilization and cell damage via providing the link between activation of glutamate receptors and the intracellular cascade of events. Those findings explain the appearance of delayed cell death and secondary energy failure, suggesting that the inhibition of oxidative stress may be a potential therapeutic for HIE [11].

Danshen (Radix salvia miltiorrhiza root), an annual sage plant, is among the most popular medicinal herbs used in China, whose extract as an antihypertensive and a sedative is widely used for the treatment of cardiovascular and cerebrovascular diseases in recent years [12-14]. The water-soluble Danshen extracts contain several diterpene quinine analogs including tanshinone I (TsI), tanshinone II (TsII), cryptotanshinone (CTs) and dihydrotanshinone I (DTsI) [15], which are able to penetrate the blood-brain barrier [16]. Given its antioxidantive and anti-inflammatory effects both in vitro [17-19] and in vivo [19-21], tanshinone is of particular therapeutic interest in hypoxic-ischemic brain damage (HIBD). Indeed, both TsI and TsII including TsIIA and TsIIB, have obvious potential for neuroprotection against hypoxia-ischemia injury in adult mice and rat [22-24]. In addition, treatment with TsIIA significantly reduced the severity of brain injury induced by hypoxia-ischemia in the immature rat $[25,26]$. However, there is no any report on the influence of TsI on motor and cognitive functions in neonatal rat after HIBD.

As aforementioned, TsI has potential anti-oxidative activity. We therefore hypothesize that TsI may present neuroprotective effects through suppressing oxidative stress, and subsequently improve motor function and learning and memory ability in neonatal rat model of HIBD. In the present study, we investigated this hypothesis using a combination of behavioral test, immunohistochemical and anti-oxidative activity analysis in the neonatal rat model of HIBD.

\section{Methods}

\section{Experimental animals}

Unsexed 7-day-old Sprague-Dawley (SD) rats were used to establish the HIBD model as previously described, with modification [27]. Briefly, the left carotid artery of 7 -day postnatal rats was ligated and $2 \mathrm{~h}$ later the pups were exposed to hypoxic conditions $\left(8 \% \mathrm{O}_{2}+92 \% \mathrm{~N}_{2}\right)$ for $2.5 \mathrm{~h}$ at $37^{\circ} \mathrm{C}$. The sham animals were only separated out the left carotid artery without ligation and no exposure to hypoxic conditions. Immediately after hypoxic treatment, pups were transferred back to the nest with their dam until weaning on postnatal day 21, and they were housed in plastic cages with unlimited access to food and water and maintained in a temperaturecontrolled colony room $\left(21{ }^{\circ} \mathrm{C}\right)$ under a cycle of 12 -h light/12-h dark (8:00 am - 8:00 pm).

\section{Tsl treatment}

To illustrate the neuroprotective effects of TsI against HIBD, all pups were divided into four groups: sham, sham + TsI, HIBD + saline, HIBD + TsI. TsI was purchased from selleck (Shanghai, China) and dissolved in sterile saline containing $0.5 \%$ dimethyl sulfoxide (DMSO). The rats in sham + TsI and HIBD + TsI groups received intraperitoneal (i.p.) injection of TsI at a dose of $5 \mathrm{mg} / \mathrm{kg} /$ day from 1 day before hypoxic-ischemic surgery (postnatal day 6) for 7 days, and the second injection was conducted 6 h before the surgery (Fig. 1a), as reported previously $[23,26]$.

To further examine the therapeutic effects of TsI against HIBD, some rats received 7-day treatment of TsI ( $5 \mathrm{mg} / \mathrm{kg} /$ day, i.p.) from postnatal day 7 to 13 , and the first injection was conducted immediately after HIBD (Fig. 3a).

\section{Grasping test}

Grasping test was used to evaluate myodynamia according to the instruction (Chatillon, USA). Briefly, 3 weeks after HIBD model established, the left and right forelimbs of each rat were placed on the grasping force sensor, respectively. Gently pulled the trail and recorded the last maximum tensile force until the rat cannot hold on. The myodynamia of left or right forelimb was measured 5 times and mean value was calculated to illustrate the myodynamia of the rats. The Grip Strength Meter was cleaned with 70\% ethanol and water between tests.

\section{Rotarod test}

Rotarod test was used to evaluate motor performance as previously described, with modifications [28]. Briefly, after grasping test, each rat received 2 rounds of pretraining trials (one trial at $0 \mathrm{rpm}$ and the other trial at $20 \mathrm{rpm}$ ) on the rotarod (Stoelting Co.). Twenty-four hours after the pre-training, rats were received formal rotarod test in ten consecutive trials (20-min intervals) with an initial rotation of $5 \mathrm{rpm}$ to last rotation $50 \mathrm{rpm}$ (increasing by $5 \mathrm{rpm}$ each time). The time that the animals remained on the rotarod during each test was monitored, and maximum test time (cut-off limit) was $180 \mathrm{~s}$. The latency to fall off the rotarod was used to express the motor performance. The rotarod was cleaned with $70 \%$ ethanol and water between tests.

\section{The Morris water maze test}

Four weeks after HIBD, the Morris water maze test was performed to measure spatial learning and memory as 

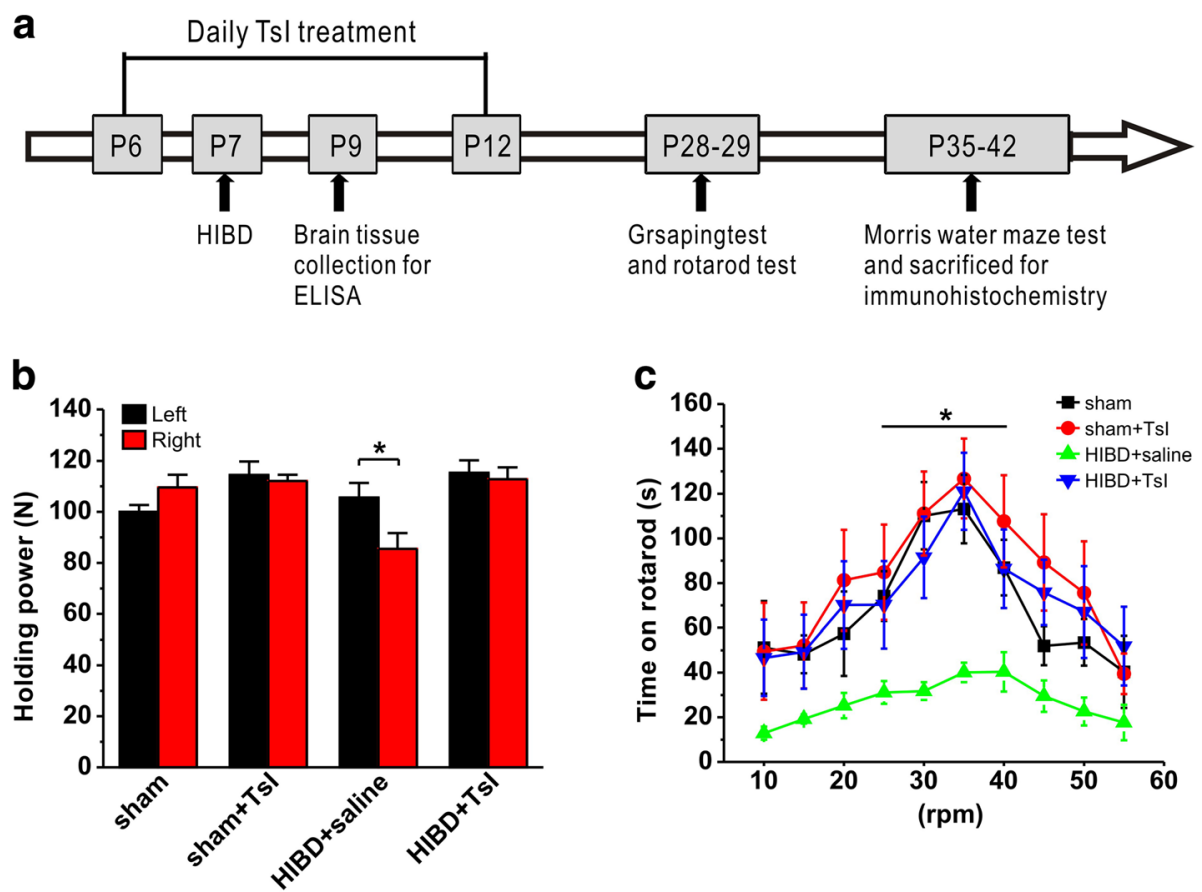

Fig. 1 Pretreatment of Tsl rescues the deficits of myodynamia and motor functions in neonatal HIBD rats. a The flowchart illustrates the experimental protocols of Tsl treatments and behavioral tests. Rats received Tsl treatment ( $5 \mathrm{mg} / \mathrm{kg}$ per day, i.p.) twice before and five times after HIBD model establish, and the second treatment of Tsl was $6 \mathrm{~h}$ before the surgery. Two days after HIBD, brain tissue was collected for oxidative stress analysis. Grasping test and rotarod test were performed on postnatal day 28 and 29 (P28-29), and the Morris water maze test was performed from postnatal day 35 to 42 (P35-42). After behavioral tests, brain tissues were collected for immunohistochemistry. $\mathbf{b}$ The left and right forelimb myodynamia during the grasping test. $\mathbf{c}$ The latency to fall off the rod during the rotarod test. The data was presented as mean \pm SEM. ${ }^{*} p<0.05$

described previously $[29,30]$. In brief, rats were allowed to adapt to the water maze, which consists of a circular pool (180-cm diameter, $60-\mathrm{cm}$ at the side), for $60 \mathrm{~s} 24 \mathrm{~h}$ before the first spatial learning trial. During training period, rats were trained in the pool over 4 trials per day for 5 consecutive days to find a submerged platform (1- $\mathrm{cm}$ below the surface of the water). During each trial, the rats failed to reach the hidden platform within $60 \mathrm{~s}$ were guided to the platform where they remained for 30 s. A 60 -s probe test was performed without the hidden platform to assess the memory retrieval ability, $24 \mathrm{~h}$ after the last training trial. All trials were recorded by using Any-maze tracking system (Stoelting, USA).

\section{Immunohistochemistry}

After behavioral tests, the rats were deeply anesthetized with urethane $(1.5 \mathrm{~g} / \mathrm{kg}$, i.p.) and then transcardially perfused with $0.9 \%$ saline followed by $4 \%$ paraformaldehyde in $100 \mathrm{mM}$ phosphate-buffered saline (PBS, PH 7.4). Brains were transferred to $30 \%$ sucrose in $100 \mathrm{mM}$ PBS for several days before they sunk to the bottom of sucrose solution. Then they were serially sectioned into $30-\mu \mathrm{m}$ coronal sections using Leica cryostat and every sixth slice with the same reference position was stained.
After blocking and permeabilization, the slices were incubated with diluted anti-NeuN (1:100 dilution, Millipore, MAB377) for overnight at $4{ }^{\circ} \mathrm{C}$. Thereafter, the positive neurons were visualized with anti-mouse Ig HRP detection kit according to the manufacturer's instruction. The number of NeuN-positive cells was quantified in brain sections with typical morphology of the pyramidal neuron in the CA1 area of hippocampus. Briefly, the number of NeuN-immunoreactive neurons was counted manually at five-section intervals throughout the CA1 area of hippocampus by bright-field microscopy using Image software. To quantify changes of the number of NeuN-immunoreactive neurons in the CA1, the number of NeuN-immunoreactive neurons in sham rat was normalized to $100 \%$, and the number of NeuN-immunoreactive neurons in other groups was expressed as a percentage of the sham.

\section{Oxidative stress analysis}

Brain tissues of hippocampus and cortex were homogenized with saline (tissue weight: saline volume $=1$ : 9) on ice, followed by centrifugation at $2500 \mathrm{~g}$ for $10 \mathrm{~min}$ at $4{ }^{\circ} \mathrm{C}$. The supernatant was used to test the antioxidant activity including total antioxidant capacity (T-AOC), glutathione (GSH), superoxide dismutase (SOD) and 
catalase (CAT), and pro-oxidants including hydrogen peroxide $\left(\mathrm{H}_{2} \mathrm{O}_{2}\right)$, total nitric oxide synthase (TNOS) and inducible nitric oxide synthase (iNOS), by using ELISA kit (Nanjing Jiancheng Bioengineering Institute, Nanjing, China). Briefly, the supernatant of brain tissue homogenate was mixed with Reagent 1 (the volume of supernatant: Reagent $1=1: 1$ ) by vortex, and then centrifuged at $3500 \mathrm{~g}$ at $4{ }^{\circ} \mathrm{C}$ for $10 \mathrm{~min}$. Then, $100 \mu \mathrm{l} \mathrm{mixed} \mathrm{tissue}$ sample was pipetted into a well of microplate, and $100 \mu \mathrm{l}$ Reagent 2 and $25 \mu \mathrm{l}$ Reagent 3 were added to each well and incubated at room temperature for $5 \mathrm{~min}$. The absorbance was determined at $405 \mathrm{~nm}$ by microplate reader (Bio Tek Insruments). Finally, the standard curve was used to determine the concentration of each sample.

\section{Statistical analysis}

All data were expressed as means \pm standard error (mean \pm SEM). The differences of rotarod test and spatial learning in water maze test among different groups were analyzed by two-way AVOVA with treatment (group) as the between-subjects factor and training trials in rotarod test or learning day in water maze test as the withinsubjects factor. The data of all other experiments were analyzed by one-way ANOVA followed by Tukey's post hoc test. Significance level was set as at $p<0.05$.

\section{Results}

Tsl ameliorates myodynamia and motor deficit in HIBD rat

To determine whether TsI can rescue HIBD-induced motor deficits, two different behavioral tests were introduced: grasping test and rotarod test. In grasping test, myodynamia of the right forelimb was significantly decreased compared to that of the left in HIBD rats (HIBD + saline: $n=10$, Fig. 1b). Importantly, treatment with TsI $(5 \mathrm{mg} / \mathrm{kg}$, i.p.) fully rescued the HIBD-induced myodynamia deficit, as reflected by similar myodynamia in both left and right forelimbs (HIBD + TsI: $n=11$, Fig. 1b). Notably, myodynamia was not affected with or without TsI treatment in sham groups (sham: $n=10$; sham + TsI: $n=10$, Fig. 1b).

In rotarod test, the rats in HIBD group spent much less time on the rod compared with those treated with sham surgery (sham: $n=8$; HIBD + saline: $\mathrm{n}=8, p<0.05$ vs. sham; Fig. 1c), indicating a significant impairment of motor balance and coordination. Treatment with TsI fully rescued the HIBD-induced motor deficit, as reflected by a dramatic increase in the time spent on the
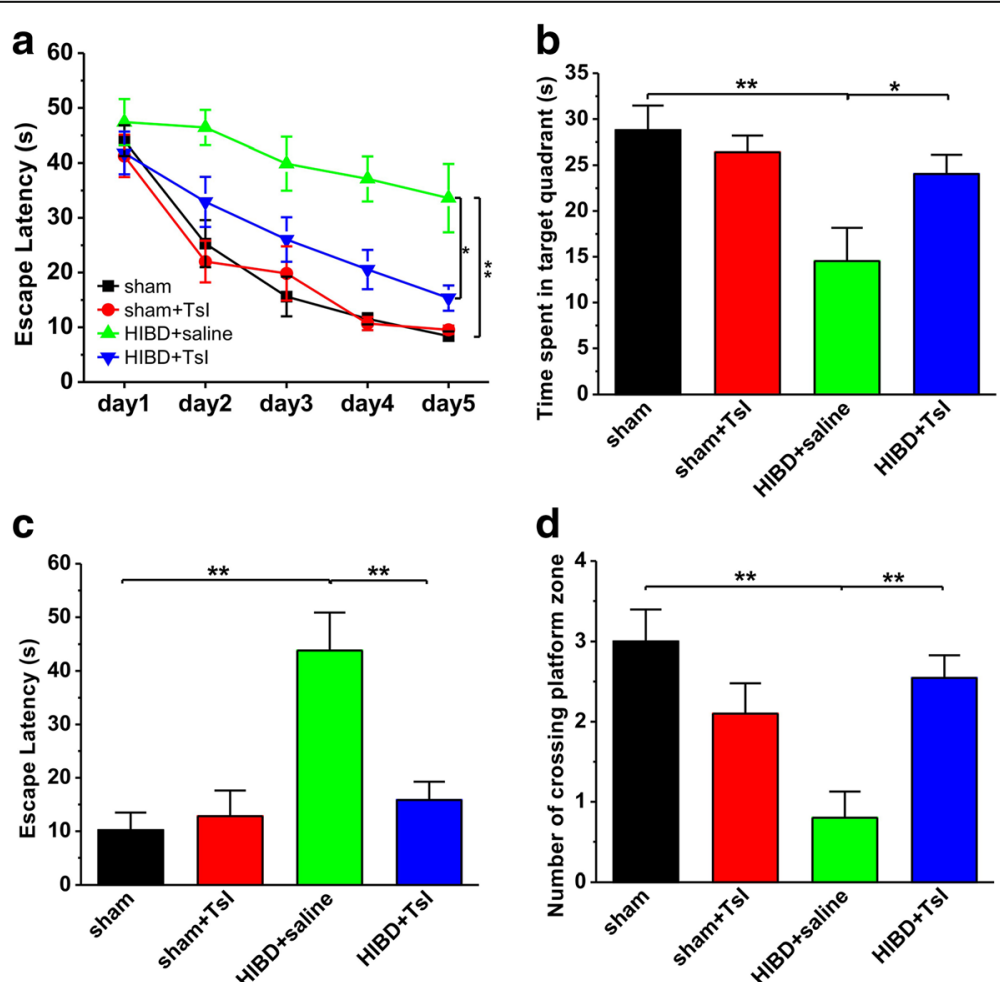

Fig. 2 Pretreatment of Tsl alleviates spatial learning and memory deficits in neonatal HIBD rats. a The average escape latency to the hidden platform location is plotted for each spatial learning day in the Morris water maze task. b-d Bar graph showed the time spent in the hidden platform-located quadrant $\mathbf{b}$, the latency of first time to the hidden platform location (c) and the number of entries into the platform zone (d) during the probe test with absence of the hidden platform, which is conducted $24 \mathrm{~h}$ after the last learning trial. Data was expressed as mean \pm SEM. ${ }^{*} p<0.05,{ }^{* *} p<0.01$ 
rod (HIBD + TsI: $n=9, p>0.05$ vs. sham, $\mathrm{p}<0.05$ vs. HIBD + saline; Fig. 1c), whereas TsI treatment have no effect on motor function in the sham group (sham + TsI: $\mathrm{n}=8, \mathrm{p}>0.05$ vs. sham; Fig. 1c). Taken together, these results indicate that TsI treatment alleviates the deficits of myodynamia and motor function in the neonatal rat after HIBD.

Tsl ameliorates spatial learning and memory in HIBD rats It has been well documented that the impairment of learning and memory is the major sequelae of HIBD in human and a variety of animal models [31, 32]. To identify the effects of TsI treatment on spatial learning and memory deficits induced by hypoxia-ischemia in neonatal rats, the Morris water maze test, a hippocampus-dependent task, was conducted. As shown in Fig. 2, although the escape latency decreased progressively in all groups, the latency in the HIBD group was much longer than that in the sham groups during spatial training period (sham: $n=10$; sham + TsI: $n=10, p>$ 0.05 vs. sham; HIBD + saline: $\mathrm{n}=10, p<0.01$ vs. sham; Fig. 2a), indicating an impairment of learning after HIBD. Importantly, daily TsI treatment $(5 \mathrm{mg} / \mathrm{kg}$, i.p.) significantly ameliorated the impairment, as reflected

a

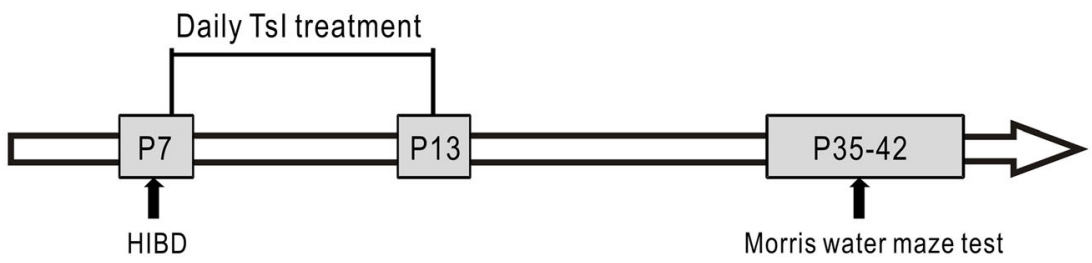

b

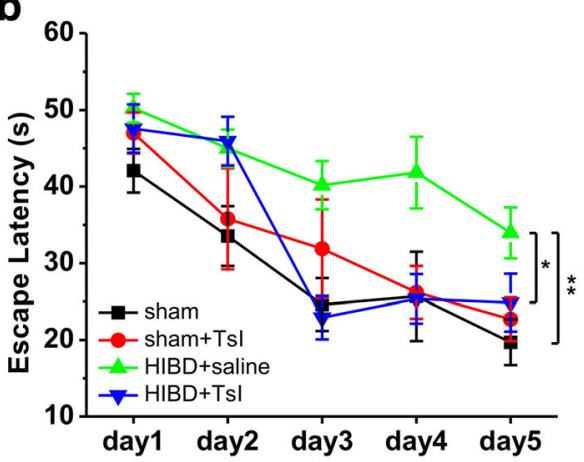

d

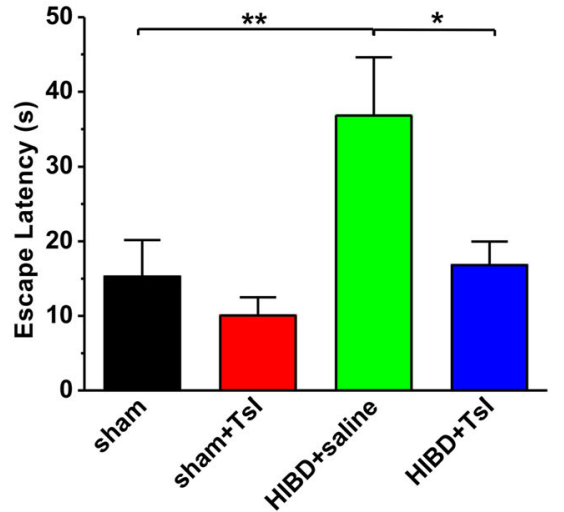

C

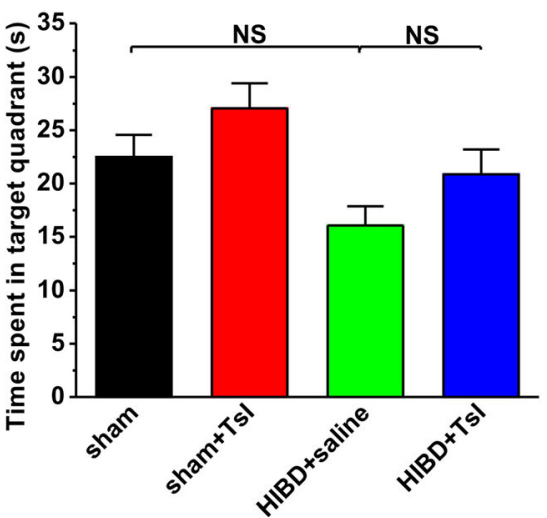

e

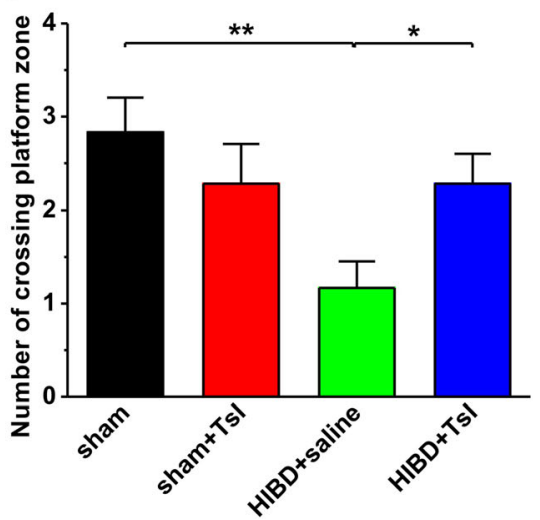

Fig. 3 Tsl treatment immediately after HIBD alleviates spatial learning and memory deficits in neonatal HIBD rats. a The flowchart illustrates the experimental protocols of Tsl treatments and water maze test. Rats received Tsl treatment (5 mg/kg per day, i.p.) seven times after HIBD model established, and the first treatment of TsI was conducted immediately after HIBD. The Morris water maze test was performed from postnatal day 35 to 42 (P35-42). b The average escape latency to the hidden platform location is plotted for each spatial learning day in the Morris water maze task. c-e Bar graph showed the time spent in the hidden platform-located quadrant $\mathbf{c}$, the latency of first time to the hidden platform location (d) and the number of entries into the platform zone (e) during the probe test with absence of the hidden platform, which is conducted $24 \mathrm{~h}$ after the last learning trial. Data was expressed as mean \pm SEM. ${ }^{*} p<0.05,{ }^{* *} p<0.01$ 
by an obvious decrease in the latency to the platform, compared with saline treatment (HIBD + TsI: $n=11, p<$ 0.05 vs. HIBD + saline; Fig. $2 a$ ).

The results from probe test showed that spatial memory retrieval was obviously impaired in HIBD rats since they spent much less time in the quadrant where the hidden platform was previously located (sham: $n=10,28.8 \pm 2.7 \mathrm{~s}$; sham + TsI: $n=10,26.4 \pm 1.8 \mathrm{~s}, p>0.05$ vs. sham; HIBD + saline: $n=10,14.5 \pm 3.6 \mathrm{~s}, p<0.01$ vs. sham; Fig. $2 \mathrm{~b})$. As expected, TsI treatment significantly increased the time spent in the target quadrant compared with the HIBD group (HIBD + TsI: $n=11,24.0 \pm 2.1 \mathrm{~s}, \mathrm{p}<0.05$ vs. HIBD + saline, $p>0.05$ vs. sham; Fig. 2b). Additionally, the results of latency to cross the location of hidden platform (sham: $n=10,10.2 \pm 3.3 \mathrm{~s}$; sham + TsI: $\mathrm{n}=10,12.8 \pm 4.8 \mathrm{~s}, p>0.05$ vs. sham; HIBD + saline: $n=10,43.8 \pm 7.0 \mathrm{~s}, p<0.01$ vs. sham; HIBD + TsI: $n=11,15.8 \pm 3.4 \mathrm{~s}, p>0.05$ vs. sham, $p<0.01$ vs. HIBD + saline; Fig. 2c) and the number of crossing the location of hidden platform (sham: $n=10,3.0 \pm$ 0.4 ; sham + TsI: $n=10,2.1 \pm 0.4, p>0.05$ vs. sham; HIBD + saline: $n=10,0.8 \pm 0.3, p<0.01$ vs. sham; HIBD + TsI: $n=11,2.5 \pm 0.3, p>0.05$ vs. sham, $p<0.01$ vs. HIBD + saline; Fig. 2d) further confirmed that spatial memory retrieval was impaired after HIBD, and TsI treatment succeeded in preventing this impairment.

To further determine the therapeutic effects of TsI on HIBD, we treated the rats with TsI daily for 7 days and the first injection was carried out immediately after HIBD. Four weeks after HIBD, the Morris water maze test was performed. As shown in Fig. 3, the protective effects of TsI on spatial learning and memory in HIBD rats were similar to those that found in pretreatment of TsI, as reflected by an obvious decrease in the latency to the platform, compared with saline treatment (HIBD + TsI: $n=7, p<0.05$ vs. HIBD + saline; Fig. 3b) during spatial training period. Although the time spent in the target quadrant among these groups was no significant difference (sham: $n=6,22.5 \pm 2.1 \mathrm{~s}$; sham + TsI: $n=7$, $27.1 \pm 2.4 \mathrm{~s}, p>0.05$ vs. sham; HIBD + saline: $n=6,16.1$ $\pm 1.8 \mathrm{~s}, p>0.05$ vs. sham; HIBD + TsI: $n=7,20.9 \pm 2.3 \mathrm{~s}$, $p>0.05$ vs. sham, $p>0.05$ vs. HIBD + saline; Fig. 3c), the latency to cross the location of hidden platform (sham: $n=6,15.3 \pm 4.9 \mathrm{~s} ;$ sham + TsI: $n=7,10.1 \pm 2.4 \mathrm{~s}, p>0.05$ vs. sham; HIBD + saline: $n=6,36.8 \pm 7.8 \mathrm{~s}, p<0.01$ vs. sham; HIBD + TsI: $n=7,16.8 \pm 3.2 \mathrm{~s}, p>0.05$ vs. sham, $p<0.05$ vs. HIBD + saline; Fig. $3 \mathrm{~d}$ ) and the number of crossing the location of hidden platform (sham: $n=6$, $2.8 \pm 0.4$; sham + TsI: $\mathrm{n}=7,2.3 \pm 0.4, p>0.05$ vs. sham; HIBD + saline: $n=6,1.2 \pm 0.3, p<0.01$ vs. sham; HIBD + TsI: $n=7,2.3 \pm 0.3, p>0.05$ vs. sham, $p<0.05$ vs. HIBD + saline; Fig. 3e) showed that spatial memory retrieval was impaired after HIBD, and TsI treatment succeeded in preventing this impairment.
Taken together, these results suggest that systemic administration of TsI either pretreatment or immediately after HIBD is able to prevent the HIBD-induced impairment of spatial learning and memory.

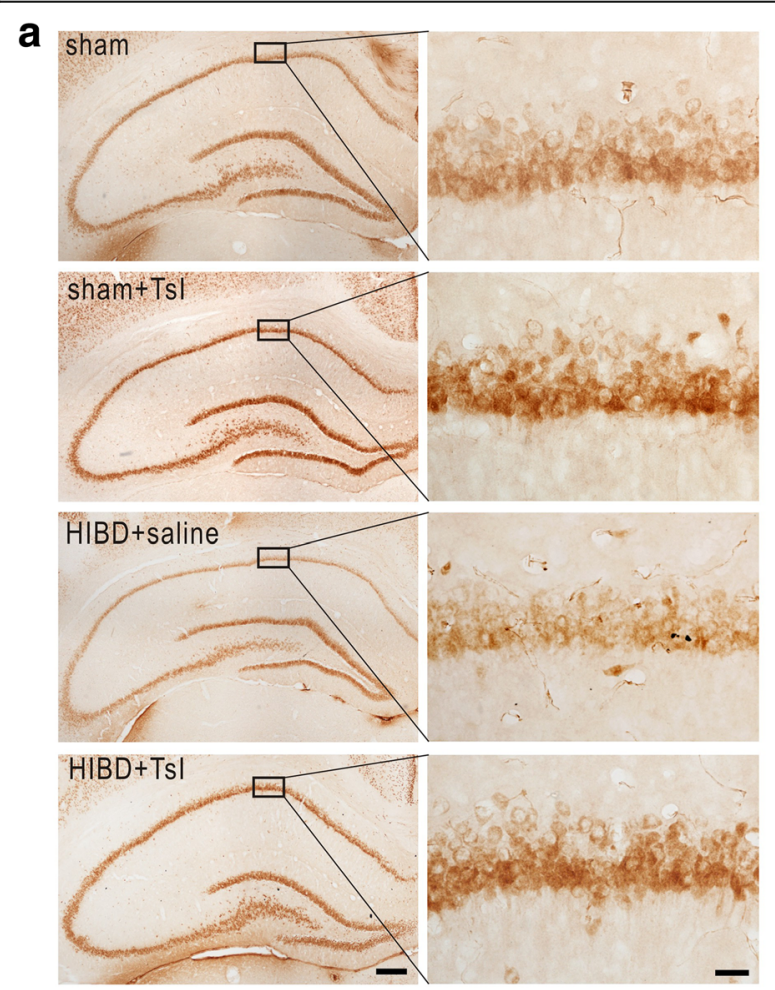

b

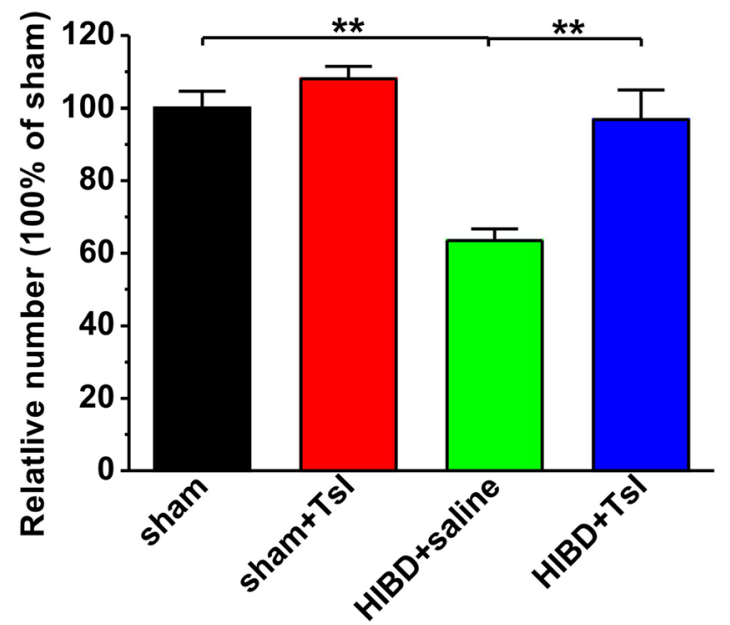

Fig. 4 Pretreatment of Ts। rescues the decrease of NeuNimmunoreactive neurons in the hippocampal CA1 region of neonatal HIBD rats. a Representative photomicrographs of different treatment. Scale bar $=400 \mu \mathrm{m}$ for left panel and $50 \mu \mathrm{m}$ for right panel. b Bar graph summarizing the relative number of NeuNimmunoreactive neurons in the CA1 region of hippocampus. Data was expressed as mean \pm SEM. ${ }^{* *} p<0.01$ 
TsI reduces HIBD-induced neuron loss

The number of NeuN-immunoreactive neurons was examined to confirm the neuroprotective effects of TsI on pyramidal neurons after hypoxic-ischemic insult in the CA1 area of hippocampus. The results showed that the number of NeuN-immunoreactive neurons in the CA1 area dramatically decreased in the HIBD group compared with the sham group, and administration of TsI significantly suppressed the decrease of NeuN-immunoreactive neurons (sham: $n=4$; sham + TsI: $n=4$, $108.0 \pm 3.5 \%$ sham, $p>0.05$ vs. sham; HIBD + saline: $n=6$, $63.5 \pm 3.2 \%$ sham, $p<0.01$ vs. sham; HIBD + TsI: $n=5$, $96.9 \pm 8.1 \%$ sham, $p>0.05$ vs. sham, $p<0.01$ vs. HIBD + saline; Fig. 4).

TsI reverses the decreased antioxidant and the increased pro-oxidant in HIBD rats

It has been well known that oxidative injury plays an important role in pathogenesis of HIE [6], and TsI has been shown to exert antioxidant action in various experimental models both in vitro $[33,34]$ and in vivo $[35,36]$. We therefore wanted to determine whether the reduction of neuron loss in HIBD rats treated with TsI could be attributed to its suppressing effect on oxidative stress. As shown in Fig. 5, a significant decrease in the production of antioxidants including T-AOC, GSH, CAT and T-
SOD was observed in HIBD group. However, compared with saline treatment, TsI dramatically rescued the $\mathrm{T}$ AOC (sham: $n=11$, sham + TsI: $\mathrm{n}=11,99.5 \pm 2.3 \%$ sham, $p>0.05$ vs. sham; HIBD + saline: $n=12,86.9 \pm$ $2.1 \%$ sham, $p<0.05$ vs. sham; HIBD + TsI: $n=13,99.4 \pm$ $2.5 \%$ sham, $p>0.05$ vs. sham, $\mathrm{p}<0.05$ vs. HIBD + saline; Fig. 5a), GSH (sham: $n=8$, sham + TsI: $\mathrm{n}=8,110.2 \pm$ $13.3 \%$ sham, $p>0.05$ vs. sham; HIBD + saline: $\mathrm{n}=8$, $55.8 \pm 5.2 \%$ sham, $p<0.01$ vs. sham; HIBD + TsI: $\mathrm{n}=8$, $90.1 \pm 6.3 \%$ sham, $p>0.05$ vs. sham, $\mathrm{p}<0.01$ vs. HIBD + saline; Fig. $5 \mathrm{~b}$ ), CAT (sham: $n=9$, sham + TsI: $n=10$, $105.1 \pm 9.8 \%$ sham, $p>0.05$ vs. sham; HIBD + saline: $\mathrm{n}=$ $10,74.4 \pm 3.7 \%$ sham, $p<0.01$ vs. sham; HIBD + TsI: $n=$ $12,94.0 \pm 3.5 \%$ sham, $p>0.05$ vs. sham, $p<0.05$ vs. HIBD + saline; Fig. $5 \mathrm{c}$ ) and T-SOD (sham: $n=8$, sham + TsI: $n=7,101.8 \pm 3.1 \%$ sham, $p>0.05$ vs. sham; HIBD + saline: $n=8, \quad 85.4 \pm 5.2 \%$ sham, $p<0.01$ vs. sham; HIBD + TsI: $n=7,107.0 \pm 4.6 \%$ sham, $p>0.05$ vs. sham, $p<0.01$ vs. HIBD + saline; Fig. 5d) activity following HIBD in the brain.

Meanwhile, as shown in Fig. 6, a significant increase in the production of pro-oxidants including $\mathrm{H}_{2} \mathrm{O}_{2}$, TNOS and iNOS was observed in HIBD group. As expected, TsI dramatically suppressed the increase of $\mathrm{H}_{2} \mathrm{O}_{2}$ (sham: $n=10$, sham + TsI: $n=10,90.9 \pm 7.9 \%$ sham, $p>0.05$ vs. sham; HIBD + saline: $n=12,132.7$
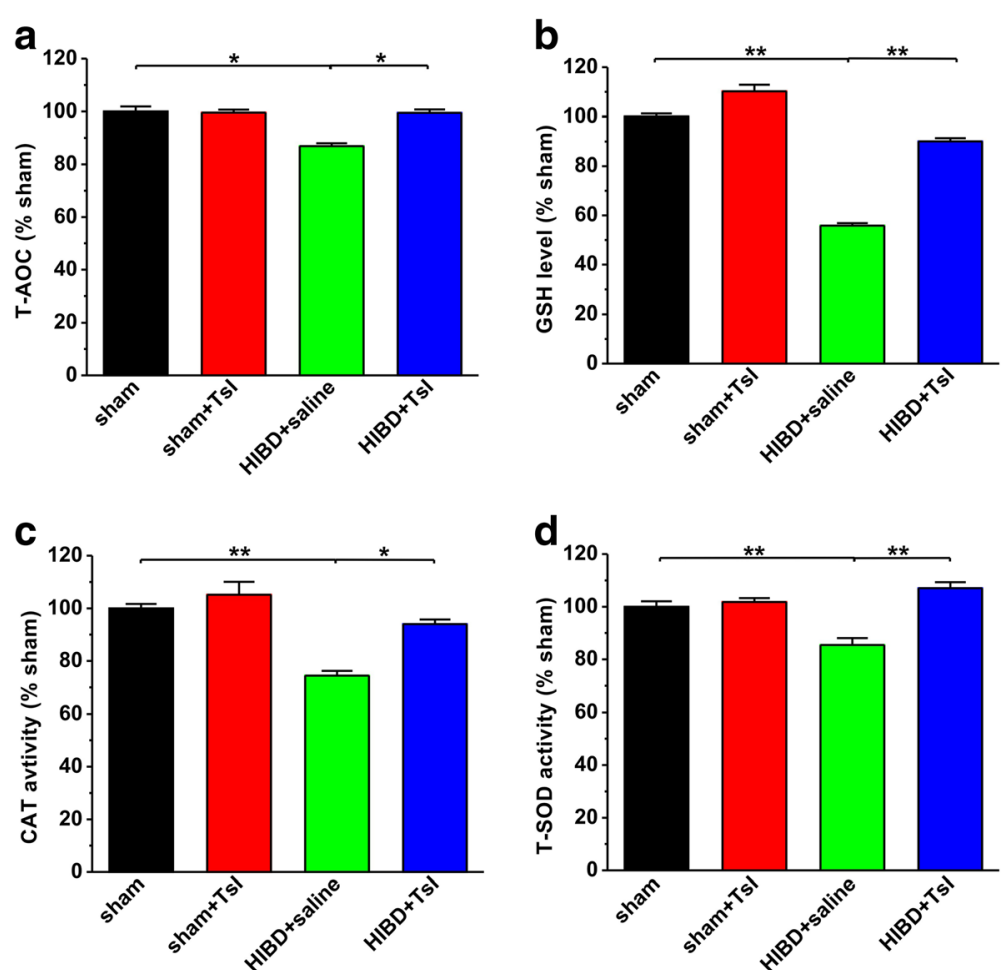

Fig. 5 Pretreatment of Tsl restores the decreased antioxidants in the brain of HIBD rats. HIBD results in a significant reduction in antioxidants including T-AOC $\mathbf{a}, \mathrm{GSH} \mathbf{b}, \mathrm{CAT} \mathbf{c}$ and T-SOD $\mathbf{d}$, whereas Tsl treatment restores these antioxidants to control level. The results were presented as the mean \pm SEM. ${ }^{*} p<0.05,{ }^{* *} p<0.01$ 


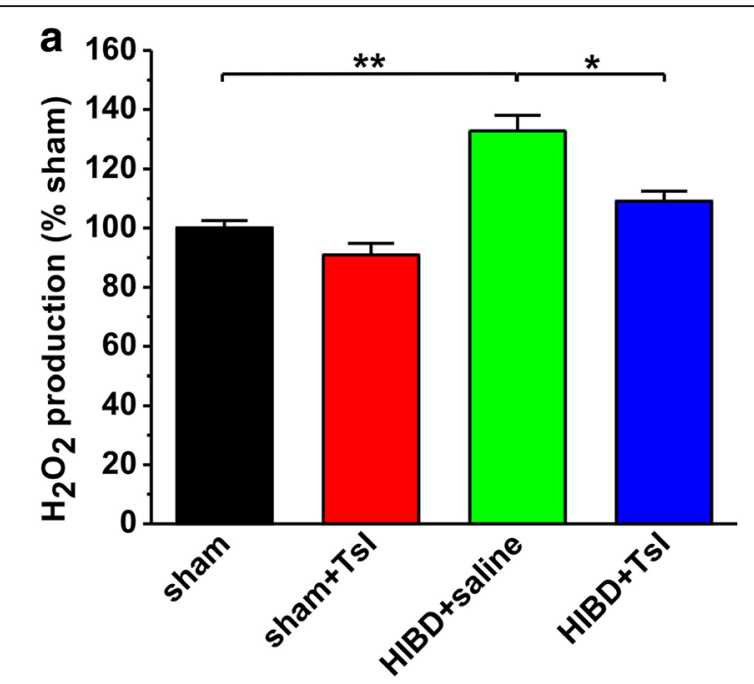

Fig. 6 Pretreatment of Tsl restores the increased pro-oxidants in the brain of HIBD rats. HIBD results in a significant increase in pro-oxidants including $\mathrm{H}_{2} \mathrm{O}_{2} \mathbf{a}$, TNOS $\mathbf{b}$ and iNOS $\mathbf{c}$, whereas Tsl treatment restores these pro-oxidants to control level. The results were presented as the mean \pm SEM. ${ }^{*} p<0.05,{ }^{* *} p<0.01$

$\pm 10.5 \%$ sham, $p<0.01$ vs. sham; HIBD + TsI: $\mathrm{n}=12$, $109.0 \pm 6.8 \%$ sham, $\mathrm{p}>0.05$ vs. sham, $\mathrm{p}<0.05$ vs. HIBD + saline; Fig. 6a), TNOS (sham: $n=11$, sham + TsI: $\mathrm{n}=9,99.4 \pm 3.6 \%$ sham, $\mathrm{p}>0.05$ vs. sham; HIBD + saline: $\mathrm{n}=11,133.0 \pm 3.7 \%$ sham, $\mathrm{p}<0.01$ vs. sham; HIBD + TsI: $n=13,108.3 \pm 5.6 \%$ sham, $\mathrm{p}>0.05$ vs. sham, $\mathrm{p}<0.05$ vs. HIBD + saline; Fig. $6 \mathrm{~b}$ ) and iNOS (sham: $\mathrm{n}=10$, sham + TsI: $\mathrm{n}=10,99.4 \pm 16.3 \%$ sham, $\mathrm{p}>0.05$ vs. sham; HIBD + saline: $\mathrm{n}=12,140.3 \pm 8.4 \%$ sham, $\mathrm{p}<0.01$ vs. sham; HIBD + TsI: $n=14,102.7 \pm$ $6.3 \%$ sham, $\mathrm{p}>0.05$ vs. sham, $P<0.01$ vs. HIBD + saline; Fig. 6c) following HIBD in the brain, compared

b

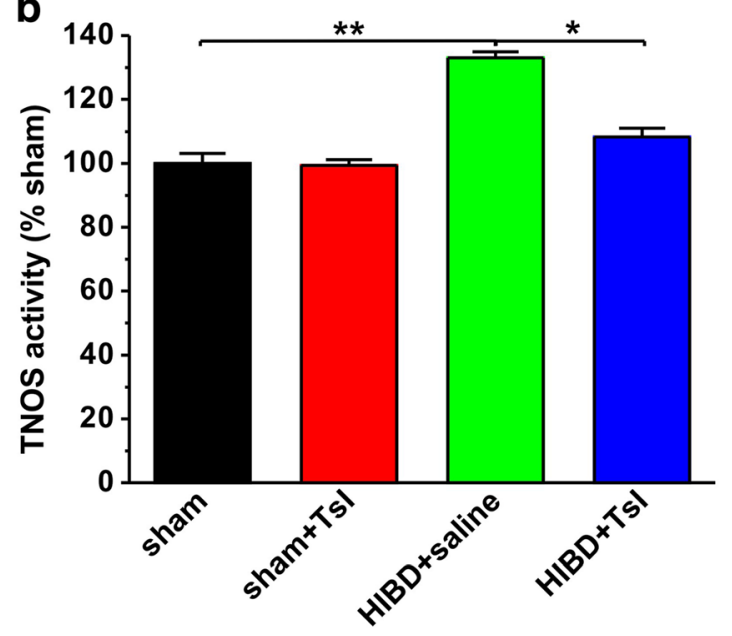

C

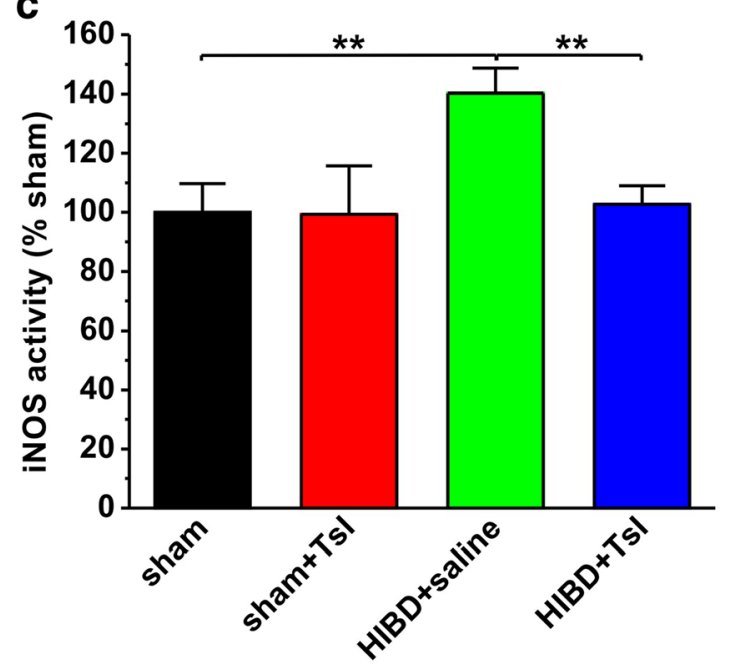
with saline treatment. These results indicate a powerful antioxidative capacity of TsI in HIBD rats.

\section{Discussion}

In the present study, we demonstrate that TsI, a compound purified from the Chinese herb Danshen, suppresses the decrease of pyramidal neurons in the CA1 area of hippocampus, and alleviates the impairment of motor and spatial learning and memory functions in the neonatal rats subjected to HIBD. We further confirm that the beneficial effects of TsI on HIBD rats are associated with the increase of antioxidant activity. We have therefore provided evidence that TsI may suppress HIBD-induced oxidative stress and subsequently block the loss of pyramidal neurons in the CA1 area of hippocampus, thereby ameliorating motor and cognitive decline.

The dried root of Salvia miltiorrhiza, called Danshen in China, has been used effectively for the treatment of many diseases, especially ischemic cardiovascular disease in aging people for over 1000 years [12]. Previous study has indicated that Danshen is able to dilate cerebral arteries, increase cerebral blood flow, inhibit oxidative stress and reduce neuronal death induced by ischemia [14]. In addition, a growing body of evidence has shown that the major lipophilic diterpenes derived from Danshen extract, such as CTs, DTsI, TsI, TsIIA and TsIIB, have neuroprotective effects against transient ischemic damage in different adult animal models including gerbils [35], rats [24] and mice [22, 23]. Recent studies have reported that TsIIA treatment is significantly protective for hypoxia-ischemia in the immature rat $[25,26]$. Similar to TsIIA, we here found that treatment with TsI markedly suppressed the loss of pyramidal neurons induced by hypoxia-ischemia in the CA1 region of hippocampus (Fig. 4). However, the dose of TsI we used in the present study was $5 \mathrm{mg} / \mathrm{kg} /$ day, which is much lower 
than TsIIA (10 mg/kg/day) used in previous study [26], suggesting that TsI may be more effective than TsII for treating HIBD.

The neuronal loss induced by hypoxia-ischemia is usually associated with the deficits of motor and cognitive functions both in animal models and in patients [37-39]. Excitedly, recent study has revealed that TsI is able to ameliorate the learning and memory impairments induced by a $\mathrm{GABA}_{\mathrm{A}}$ receptor agonist diazepam, or an NMDA receptor antagonist MK-801, in adult mice [40]. However, so far no study to examine the influence of TsI on motor and learning and memory functions in immature animals after hypoxia-ischemia. In the current study, we found that TsI could rescue myodynamia and motor deficits (Fig. 1), and alleviate the spatial learning and memory impairments (Figs. 2 and 3) in neonatal HIBD model rats. Combined with previous report [40], we can therefore conclude that TsI has the ability to ameliorate the learning and memory impairments in both adult and neonatal animals.

Although HIBD is a complex multifactorial disease, a growing body of evidence has shown that oxidative damage to vital cellular structures plays a critical role in the pathogenesis of brain damage in both the immature and mature nervous system [6, 41, 42]. Several studies have indicated that TsI is able to exert antioxidative and antiapoptotic effects in cellular and mouse model of Parkinson's disease (PD) $[18,19,43]$. In full agreement with these findings, we here found that treatment with TsI markedly reversed the decrease of antioxidants and the increase of pro-oxidants induced by HIBD in neonatal rats (Figs. 5 and 6). The exact underlying cellular and molecular mechanisms remain to be determined, but may be at least in part due to the up-regulating effects of TsI on nuclear factor erythroid-2-related factor 2 (Nrf2), since previous studies have supported that the antioxidation of TsI is involved in Nrf2 signaling pathway both in vitro and in mouse model of PD [43, 44]. Thus, future experiments examining Nrf2 signaling pathway in neonatal HIBD animals with or without TsI treatment will help determine whether the therapeutic effects of TsI in neonatal HIBD rats can be attributed to its up-regulation of Nrf2. Furthermore, some studies have reported that the activation of nuclear factor kappa B (NF-kB) promotes cell survival via attenuating reactive oxygen species (ROS), such as NOS, $\mathrm{H}_{2} \mathrm{O}_{2}$, et al. $[45,46]$, and increasing the expression of antioxidant proteins, such as GSH, SOD, catalase, and so on [47-49]. Here, we found that TsI significantly promoted the expression of antioxidant proteins and suppressed the expression of pro-oxidants (Figs. 5 and 6). However, further studies would be necessary to elucidate the full mechanism whether TsI produces neuroprotection via activating NF$\kappa B$ signaling pathway.

\section{Conclusions}

In summary, these data provide the first evidence that TsI treatment suppresses HIBD-induced neuronal death and oxidative stress, thereby ameliorating myodynamia and motor abilities as well as spatial learning and memory in neonatal rats after HIBD, an animal model of HIE in patient, suggesting that TsI may represent a potentially effective therapeutic drug for HIE.

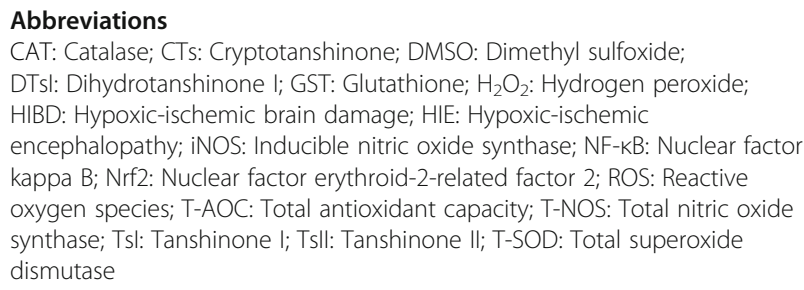

\section{Acknowledgements}

We thank Dr. Yu Tian Wang from the University of British Columbia for discussion and support.

\section{Funding}

This work was supported by grants from the National Natural Science Foundation of China (Grant No. 81571042 and 81,622,015), the National Basic Research Program of China (Grant No. 2014CB548100) and Graduate student Innovation Project of Chongqing (Grant No. CYB17103).

\section{Availability of data and materials}

Data sharing not applicable to this article as no datasets were generated or analysed during the current study.

\section{Authors' contributions}

$C D$ carried out the behavioral and immunohistochemical studies, and drafted the manuscript. YL carried out the ELISA assays. ZD conceived of the study, and participated in its design and coordination and helped to draft the manuscript. All authors read and approved the final manuscript.

\section{Ethics approval and consent to participate}

All procedures were performed in accordance with Chongqing Science and Technology Commission guidelines for animal research and approved by the Chongqing Medical University Animal Care Committee, and every effort was made to minimize both the animal suffering and the number of animals used.

\section{Consent for publication}

Not applicable.

Competing interests

The authors have declares that they have no competing interests.

\section{Publisher's Note}

Springer Nature remains neutral with regard to jurisdictional claims in published maps and institutional affiliations.

\section{Author details}

${ }^{1}$ Ministry of Education Key Laboratory of Child Development and Disorders, Children's Hospital of Chongqing Medical University, 136 Zhongshan Er Road, Yuzhong District, Chongqing 400014, People's Republic of China. ${ }^{2}$ Chongqing Key Laboratory of Translational Medical Research in Cognitive Development and Learning and Memory Disorders, Children's Hospital of Chongqing Medical University, 136 Zhongshan Er Road, Yuzhong District, Chongqing 400014, People's Republic of China. ${ }^{3}$ China International Science and Technology Cooperation base of Child Development and Critical Disorders, Children's Hospital of Chongqing Medical University, 136 Zhongshan Er Road, Yuzhong District, Chongqing 400014, People's Republic of China. 
Received: 17 September 2017 Accepted: 7 November 2017 Published online: 14 November 2017

\section{References}

1. Kurinczuk JJ, Whitekoning M, Badawi N. Epidemiology of neonatal encephalopathy and hypoxic-ischaemic encephalopathy. Early Hum Dev. 2010;86(6):329-38

2. Graham EM, Ruis KA, Hartman AL, Northington FJ, Fox HE. A systematic review of the role of intrapartum hypoxia-ischemia in the causation of neonatal encephalopathy. Am J Obstet Gynecol. 2008;199(6):587-95.

3. Jacobs SE, Morley CJ, Inder TE, Stewart MJ, Smith KR, McNamara PJ, Wright IM, Kirpalani HM, Darlow BA, Doyle LW. Infant Cooling Evaluation Collaboration. Whole-body hypothermia for term and near-term newborns with hypoxic-ischemic encephalopathy: a randomized controlled trial. Arch Pediatr Adolesc Med. 2011;165(12):692-700.

4. Martinezbiarge M, Diezsebastian J, Kapellou O, Gindner D, Allsop JM Rutherford MA, Cowan FM. Predicting motor outcome and death in term hypoxic-ischemic encephalopathy. Neurology. 2011;76(24):2055-61.

5. Shankaran S, Pappas A, McDonald SA, Vohr BR, Hintz SR, Yolton K, Gustafson KE, Leach TM, Green C, Bara R, Petrie Huitema CM, Ehrenkranz RA, Tyson JE, Das A, Hammond J, Peralta-Carcelen M, Evans PW, Heyne RJ, WilsonCostello DE, Vaucher YE, Bauer CR, Dusick AM, Adams-Chapman I, Goldstein RF, Guillet R, Papile LA, Higgins RD; Eunice Kennedy Shriver NICHD Neonatal Research Network. Childhood outcomes after hypothermia for neonatal encephalopathy. N Engl J Med. 2012;366(22):2085-92.

6. Ferriero DM. Oxidant mechanisms in neonatal hypoxia-ischemia. Dev Neurosci. 2001;23(3):198-202

7. Halliwell B. Reactive oxygen species and the central nervous system. J Neurochem. 1992;120(5):1609-23.

8. Lafemina MJ, Sheldon RA, Ferriero DM. Acute hypoxia-ischemia results in hydrogen peroxide accumulation in neonatal but not adult mouse brain. Pediatr Res. 2006;59(5):680-3.

9. Wallin C, Puka-Sundvall M, Hagberg H, Weber SG, Sandberg M. Alterations in glutathione and amino acid concentrations after hypoxia-ischemia in the immature rat brain. Brain Res Dev Brain Res. 2000;125(1-2):51-60.

10. Khan JY, Black SM. Developmental changes in murine brain antioxidant enzymes. Pediatr Res. 2003;54(1):77-82.

11. Guan J, Gunn AJ, Sirimanne ES, Tuffin J, Gunning MI, Clark R, Gluckman PD. The window of opportunity for neuronal rescue with insulin-like growth factor-1 after hypoxia-ischemia in rats is critically modulated by cerebral temperature during recovery. J Cereb Blood Flow Metab. 2000;20(3):513-9.

12. Ji $X Y$, Tan $K H$, Zhu YZ. Salvia miltiorrhiza and ischemic diseases. Acta Pharmacol Sin. 2000;21(12):1089-94.

13. Wu B, Liu M. Cochrane systematic review: Danshen agents for acute Ischaemic stroke. J Evid Based Med. 2005;5(2):101-5.

14. Han JY, Fan JY, Horie Y, Miura S, Cui DH, Ishii H, Hibi T, Tsuneki H, Kimura I. Ameliorating effects of compounds derived from salvia miltiorrhiza root extract on microcirculatory disturbance and target organ injury by ischemia and reperfusion. Pharmacol Ther. 2008;117(2):280-95.

15. Gu M, Zhang S, Su Z, Chen Y, Ouyang F. Fingerprinting of salvia miltiorrhiza Bunge by non-aqueous capillary electrophoresis compared with high-speed counter-current chromatography. J Chromatogr A. 2004;1057(1):133-40.

16. Ren Y, Houghton PJ, Hider RC, Howes MJ. Novel diterpenoid acetylcholinesterase inhibitors from salvia miltiorhiza. Planta Med. 2004;70(03):201-4

17. Zhang XS, Ha S, Wang XL, Shi YL, Duan SS, Li ZA. Tanshinone IIA protects dopaminergic neurons against 6-hydroxydopamine-induced neurotoxicity through miR-153/NF-E2-related factor 2/antioxidant response element signaling pathway. Neuroscience. 2015;303:489-502.

18. de Oliveira MR, Fürstenau CR, de Souza IC, da Costa Ferreira G. Tanshinone I attenuates the effects of a challenge with $\mathrm{H}_{2} \mathrm{O} 2$ on the functions of tricarboxylic acid cycle and respiratory chain in SH-SY5Y cells. Mol Neurobiol. 2016; [Epub ahead of print].

19. Xu J, Wei X, Ren M, Wang L, Zhang X, Lou H. Neuroprotective effects of Tanshinone I against 6-OHDA-induced oxidative stress in cellular and mouse model of Parkinson's disease through upregulating Nrf2. Neurochem Res. 2015:41(4):779-86.

20. Qiao Z, Ma J, Liu H. Evaluation of the antioxidant potential of salvia miltiorrhiza ethanol extract in a rat model of ischemia-reperfusion injury. Molecules. 2011;16(12):10002-12.
21. Wang S, Jing H, Yang H, Liu Z, Guo H, Chai L, Hu L. Tanshinone I selectively suppresses pro-inflammatory genes expression in activated microglia and prevents nigrostriatal dopaminergic neurodegeneration in a mouse model of Parkinson's disease. J Ethnopharmacol. 2015;164:247-55.

22. Lam BY, Lo AC, Sun X, Luo HW, Chung SK, Sucher NJ. Neuroprotective effects of tanshinones in transient focal cerebral ischemia in mice. Phytomedicine. 2003;10(4):286-91.

23. Lee JC, Park JH, Park OK, Kim IH, Yan BC, Ahn JH, Kwon SH, Choi JH, Kim JD, Won $\mathrm{MH}$. Neuroprotective effects of tanshinone I from Danshen extract in a mouse model of hypoxia-ischemia. Anat Cell Biol. 2013;46(3):183-90.

24. Liu L, Zhang XL, Yang R, Cui L, Li M, Du W, Wang S. The neuroprotective effects of Tanshinone IIA are associated with induced nuclear translocation of TORC1 and upregulated expression of TORC1, pCREB and BDNF in the acute stage of ischemic stroke. Brain Res Bull. 2010;82(3):228-33.

25. Hei M, Luo Y, Zhang X, Liu F. Tanshinone lla alleviates the biochemical changes associated with hypoxic ischemic brain damage in a rat model. Phytother Res. 2011;25(12):1865-9.

26. Xia WJ, Yang M, Fok TF, Li K, Chan WY, Ng PC, Ng HK, Chik KW, Wang CC, Gu GJ, Woo KS, Fung KP. Partial neuroprotective effect of pretreatment with tanshinone IIA on neonatal hypoxia-ischemia brain damage. Pediatr Res. 2005:58(4):784-90.

27. Rice JE 3rd, Vannucci RC, Brierley JB. The influence of immaturity on hypoxic-ischemic brain damage in the rat. Ann Neurol. 1981;9(2):131-41.

28. Heldermon CD, Hennig AK, Ohlemiller KK, Ogilvie JM, Herzog ED, Breidenbach A, Vogler C, Wozniak DF, Sands S. M: development of sensory, motor and behavioral deficits in the murine model of Sanfilippo syndrome type B. PLoS One. 2007;2(8):e772.

29. Ge Y, Dong Z, Bagot R, Howland J, Phillips A, Wong T, Wang Y. Hippocampal long-term depression is required for the consolidation of spatial memory. Proc Natl Acad Sci U S A. 2010;107(38):16697-702.

30. Dong Z, Han H, Li H, Bai Y, Wang W, Tu M, Peng Y, Zhou L, He W, Wu X, Tan T, Liu M, Wu X, Zhou W, Jin W, Zhang S, Sacktor TC, Li T, Song W, Wang YT. Long-term potentiation decay and memory loss are mediated by AMPAR endocytosis. J Clin Invest. 2015;125(1):234-47.

31. Dilenge ME, Majnemer A, Shevell MI. Long-term developmental outcome of asphyxiated term neonates. J Child Neurol. 2001;16(11):781-92.

32. Golan $\mathrm{H}$, Huleihel $\mathrm{M}$. The effect of prenatal hypoxia on brain development: short- and long-term consequences demonstrated in rodent models. Dev Sci. 2006;9(4):338-49.

33. Ji K, Zhao Y, Yu T, Wang Z, Gong H, Yang X, Liu Y, Huang K. Inhibition effects of tanshinone on the aggregation of a-synuclein. Food Funct. 2016;7(1):409-16.

34. Wang Q, Yu X, Patal K, Hu R, Chuang S, Zhang G, Zheng J. Tanshinones inhibit amyloid aggregation by amyloid- $\beta$ peptide, disaggregate amyloid fibrils, and protect cultured cells. ACS Chem Neurosci. 2013:4(6):1004-15.

35. Park OK, Choi $J H$, Park JH, Kim IH, Yan BC, Ahn JH, Kwon SH, Lee JC, Kim YS, Kim M, Kang IJ, Kim JD, Lee YL, Won MH. Comparison of neuroprotective effects of five major lipophilic diterpenoids from Danshen extract against experimentally induced transient cerebral ischemic damage. Fitoterapia. 2012:83(8):1666-74.

36. Kim DH, Jeon SJ, Jung JW, Lee S, Yoon BH, Shin BY, Son KH, Cheong $\mathrm{JH}$, Kim YS, Kang SS, Ko KH, Ryu JH. Tanshinone congeners improve memory impairments induced by scopolamine on passive avoidance tasks in mice. Eur J Pharmacol. 2007:574(2):140-7.

37. Ten VS, Bradley-Moore M, Gingrich JA, Stark RI, Pinsky DJ. Brain injury and neurofunctional deficit in neonatal mice with hypoxic-ischemic encephalopathy. Behav Brain Res. 2003;145(1-2):209-19.

38. Vargha-Khadem F, Gadian DG, Mishkin M. Dissociations in cognitive memory: the syndrome of developmental amnesia. Philos Trans R Soc Lond Ser B Biol Sci. 2001;356(1413):1435-40.

39. Farkas E, Luiten PG, Bari F. Permanent, bilateral common carotid artery occlusion in the rat: a model for chronic cerebral hypoperfusion-related neurodegenerative diseases. Brain Res Rev. 2007:54(1):162-80.

40. Kim DH, Kim S, Jeon SJ, Son KH, Lee S, Yoon BH, Cheong JH, Ko KH, Ryu JH. Tanshinone I enhances learning and memory, and ameliorates memory impairment in mice via the extracellular signal-regulated kinase signalling pathway. Br J Pharmacol. 2009:158(4):1131-42.

41. Vannucci RC, Perlman JM. Interventions for perinatal hypoxic-ischemic encephalopathy. Pediatrics. 1997:100(6):1004-14.

42. Zhao M, Zhu P, Fujino M, Zhuang J, Guo H, Sheikh I, Zhao L, Li XK. Oxidative stress in hypoxic-ischemic encephalopathy: molecular mechanisms and therapeutic strategies. Int J Mol Sci. 2016;17(12):2078. 
43. de Oliveira MR, Schuck PF, Bosco SM. Tanshinone I induces mitochondrial protection through an Nrf2-dependent mechanism in Paraquat-TreatedHuman neuroblastoma SH-SY5Y cells. Mol Neurobiol. 2017;54(6):4597-608.

44. Jing X, Wei X, Ren M, Wang L, Zhang X, Lou H. Neuroprotective effects of Tanshinone I against 6-OHDA-induced oxidative stress in cellular and mouse model of Parkinson's disease through upregulating Nrf2. Neurochem Res. 2016;41(4):779-86.

45. Guo Z, Shao L, Du Q, Park KS, Geller DA. Identification of a classic cytokineinduced enhancer upstream in the human iNOS promoter. FASEB J. 2007;21(2):535-42.

46. Morris KR, Lutz RD, Choi HS, Kamitani T, Chmura K, Chan ED. Role of the NFkappaB signaling pathway and kappaB cis-regulatory elements on the IRF-1 and $\mathrm{NOS}$ promoter regions in mycobacterial lipoarabinomannan induction of nitric oxide. Infect Immun. 2003;71(3):1442-52.

47. Rojo Al, Salinas M, Martin D, Perona R, Cuadrado A. Regulation of Cu/Znsuperoxide dismutase expression via the phosphatidylinositol 3 kinase/Akt pathway and nuclear factor-kappaB. J Neurosci. 2004;24(33):7324-34

48. Xia C, Hu J, Ketterer B, Taylor JB. The organization of the human GSTP1-1 gene promoter and its response to retinoic acid and cellular redox status. Biochem J. 1996;313(Pt 1):155-61.

49. Zhou LZ, Johnson AP, Rando TA. NF kappa B and AP-1 mediate transcriptional responses to oxidative stress in skeletal muscle cells. Free Radic Biol Med. 2001;31(11):1405-16.

\section{Submit your next manuscript to BioMed Central and we will help you at every step:}

- We accept pre-submission inquiries

- Our selector tool helps you to find the most relevant journal

- We provide round the clock customer support

- Convenient online submission

- Thorough peer review

- Inclusion in PubMed and all major indexing services

- Maximum visibility for your research

Submit your manuscript at www.biomedcentral.com/submit 